\title{
VOLUMETRIC LOCKING SUPPRESSION METHOD FOR NEARLY INCOMPRESSIBLE NONLINEAR ELASTIC MULTI-LAYER BEAMS USING ANCF ELEMENTS
}

\author{
Grzegorz Orzechowski, Janusz Frączek \\ Warsaw University of Technology, Institute of Aeronautics and Applied Mechanics, Warsaw, Poland \\ e-mail: gorzech@meil.pw.edu.pl
}

\begin{abstract}
The analysis and solution of many modern flexible multibody dynamic problems require formulations that are able to effectively model bodies with nonlinear materials undergoing large displacements and deformations. The absolute nodal coordinate formulation (ANCF) in connection with a continuum-based approach is one way to deal with these systems. The main objective of this work is to extend an existent approach for the modelling of slender structures within the ANCF framework with nonlinear, nearly incompressible materials using the volumetric energy penalty technique. The main part of the study is devoted to the evaluation of multi-layer beam models and simplifications in the locking suppression method based on $F$-bar projection. The results present significantly better agreement with the reference solution for multi-layer structures built with the standard ANCF beam element as compared with the earlier implementation.
\end{abstract}

Keywords: multibody dynamics, ANCF, incompressibility, locking phenomena, multi-layer beams

\section{Introduction}

The dynamic analysis of bodies that undergo large deformations and are built with complex and nonlinear materials is a vital part of the modern computer-aided design and modelling techniques. Therefore, such features should be included in a reliable manner in the advanced multibody system (MBS) simulation software. In flexible multibody dynamics, the most frequently used method is the floating frame of reference formulation (Shabana, 1997b) that is usually limited to linear-elastic deformations. The geometrical and material nonlinearities can be included within the finite element analysis (FEA) (Bathe, 1996), however, the FEA is not perfectly compatible with the MBS (Wasfy and Noor, 2003).

The absolute nodal coordinate formulation (ANCF) proposed by Shabana (1997a) can be efficiently used within the flexible multibody dynamics. The unique characteristics of this method allow straightforward modelling of beam and plate elements using nonlinear material models. ANCF employs the slope coordinates rather than rotations to describe local orientation, which enables, among other things, representation of complicated shapes using just a few elements. Flexible ANCF bodies can exactly represent rigid body modes, including large rotations, and model large body deformations. Additionally, the ANCF beam elements may employ general constitutive formulations (in addition to the classical beam theories) for a variety of nonlinear material models, including incompressible ones. All these features cause that the ANCF is well suited for the dynamic analysis of highly flexible beam structures using nonlinear material models within the MBS framework (Shabana, 2008).

Incompressible rubber-like materials are used in many engineering and industrial applications like defence, automotive, safety and others. Consequently, reliable and effective application of the incompressible nonlinear materials in many biomechanical and engineering models is one of the 
key goals. However, commercial FEA packages restrict the work with incompressible materials to

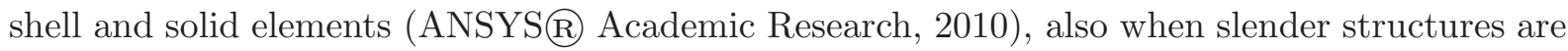
considered that are otherwise modelled with beam elements. One can overcome this limitation in the mentioned kind of common applications by applying fully parameterized ANCF beam elements (Shabana, 2008; Orzechowski and Frączek, 2015).

Most investigations devoted to the ANCF framework assume a linear-elastic material model. Compressible and incompressible hyperelastic and isotropic material models were firstly used within the ANCF by Maqueda and Shabana (2007). Furthermore, Maqueda et al. (2010) presented the application of the ANCF beams with incompressible materials to rubber chains systems. Moreover, the validation of the ANCF model based on the experiment that captured motion of the rubber-like beam was presented in (Jung et al., 2011). Nonetheless, none of the above works have stressed the importance of using the locking alleviation techniques. It is worth to point out that many issues that are actively researched in the ANCF field have already been studied for nonlinear finite elements.

The main objective of this paper is to recall and extend the volumetric locking elimination techniques for nonlinear, hyperelastic, nearly incompressible material models applied to the ANCF beams. The previous paper by Orzechowski and Frączek (2015) showed an importance of volumetric locking elimination techniques in typical applications, however, only higher-order elements, like those presented in Orzechowski and Shabana (2016), which were also numerically expensive due to the high number of coordinates and integration points, provided a reasonable results. Therefore, exemplary techniques that may reduce the computational cost are introduced and validated with several numerical examples. Strictly speaking, the implementation of multi-layer beam models with appropriate continuity between layers make it possible to use the lower-order ANCF beam element, while the use of lower-order projection basis with the $F$-bar projection technique simplifies this locking suppression formulation. Therefore, in the current study, the standard three-dimensional fully parameterized element is used (Shabana and Yakoub, 2001; Yakoub and Shabana, 2001) together with two simple incompressible material models: one-parameter Neo-Hookean and two-parameter Mooney-Rivlin (Shabana, 2008). Incompressibility of the materials is ensured by the penalty method, which is chosen due to its simple form and common use, and two methods of the locking suppression are applied. Due to introduced multi-layer structures, the results of numerical tests are in significantly better agreement with the reference for models built with the standard ANCF beam element as compared with the earlier implementation.

\section{Kinematics and dynamics of deformable bodies}

The nodal coordinates of the ANCF elements are prescribed with respect to the global reference frame and they include translational and slope coordinates. Consequently, no rotational coordinates are used to identify the element orientation. Thus, the independent rotation field interpolation is not required and only the displacement field is interpolated (Sugiyama et al., 2006).

In this investigation, the fully parameterized ANCF beam element with twenty-four nodal coordinates is used (Shabana and Yakoub, 2001; Yakoub and Shabana, 2001). Twelve nodal parameters of this beam are $\mathbf{e}^{i^{\mathrm{T}}}=\left[\begin{array}{llll}\mathbf{r}^{i \mathrm{~T}} & \mathbf{r}_{, x}^{i \mathrm{~T}} & \mathbf{r}_{, y}^{i \mathrm{~T}} & \mathbf{r}_{, z}^{i}\end{array}\right]$, where $\mathbf{e}^{i}$ is the vector of nodal coordinates of the $i$-th node, $\mathbf{r}^{i}$ is the vector of the $i$-th node global position, while $\mathbf{r}_{, k}^{i}=\partial \mathbf{r}^{i} / \partial k$ for $k=x, y, z$ are vectors of the slope coordinates of the $i$-th node. The beam element used in the study consists of two nodes, therefore, the vector of the nodal coordinates for a single-beam element is given by $\mathbf{e}^{\mathrm{T}}=\left[\mathbf{e}^{A^{\mathrm{T}}} \mathbf{e}^{B^{\mathrm{T}}}\right]$, where $A$ and $B$ indicate nodes at the beam ends. The position of an arbitrary point on the ANCF element can be obtained as follows 


$$
\mathbf{r}(\mathbf{x}, t)=\mathbf{S}(\mathbf{x}) \mathbf{e}(t)
$$

where $\mathbf{x}=\left[\begin{array}{lll}x & y & z\end{array}\right]^{\mathrm{T}}, \mathbf{S}(\mathbf{x})=\left[\begin{array}{llll}s_{1} \mathbf{I} & s_{2} \mathbf{I} & \cdots & s_{8} \mathbf{I}\end{array}\right]$ is the element shape function matrix, $\mathbf{I}$ is a $3 \times 3$ identity matrix, and

$$
\begin{array}{llll}
s_{1}=1-3 \xi^{2}+2 \xi^{3} & s_{3}=l(\eta-\xi \eta) & s_{5}=3 \xi^{2}-2 \xi^{3} & s_{7}=l \xi \eta \\
s_{2}=l\left(\xi-2 \xi^{2}+\xi^{3}\right) & s_{4}=l(\zeta-\xi \zeta) & s_{6}=l\left(-\xi^{2}+\xi^{3}\right) & s_{8}=l \xi \zeta
\end{array}
$$

where $l$ is length of the element in the undeformed state while $\xi=x / l, \eta=y / l$ and $\zeta=z / l$ are element dimensionless coordinates. It can be shown that the shape function matrix $\mathbf{S}$ can describe arbitrary rigid body motion (Yakoub and Shabana, 2001).

The mass matrix $\mathbf{M}$ of the element can be written as $\mathbf{M}=\int_{V} \rho \mathbf{S}^{\mathrm{T}} \mathbf{S} d V$, where $\rho$ and $V$ are, respectively, density and volume of the element. In the ANCF, the mass matrix is constant. In addition, the forces resulting from differentiation of kinetic energy, like Coriolis, tangential, centrifugal and others, are equal to zero. Therefore, the only nonzero quantities in the system equations of motion are the vectors of the elastic and external forces.

To derive the vector of the external forces, which comprise, for example, the gravitational forces, the principle of virtual work can be used in the form $\delta W_{e}=\mathbf{F}_{e}^{\mathrm{T}} \delta \mathbf{r}=\mathbf{F}_{e}^{\mathrm{T}} \mathbf{S} \delta \mathbf{e}=\mathbf{Q}_{e}{ }^{\mathrm{T}} \delta \mathbf{e}$, where $\delta W_{e}$ is the virtual work of the external force $\mathbf{F}_{e}$ and $\mathbf{Q}_{e}$ is the vector of the generalized external forces. For example, the nodal force vector due to gravity can be obtained as $\mathbf{Q}_{e g}^{\mathrm{T}}=\int_{V} \mathbf{F}_{e g}^{\mathrm{T}} \mathbf{S} d V$, where $\mathbf{F}_{e g}^{\mathrm{T}}=\left[\begin{array}{lll}0 & -m g & 0\end{array}\right]$ is the gravity force vector acting along the vertical axis, $m$ is total mass of an element and $g$ is the gravitational constant.

The position vector gradient of the fully parameterized ANCF element may be expressed by $\mathbf{F}=\partial \mathbf{r} / \partial \mathbf{X}$ where $\mathbf{r}$ is given by Eq. (2.1) and $\mathbf{X}=\left[\begin{array}{lll}X & Y & Z\end{array}\right]^{\mathrm{T}}$. Using directly the expression of the tensor $\mathbf{F}$, one can evaluate the value of the strain energy for an element. In the case of the linear-elastic material model, the strain energy can be written as $U_{s}=\frac{1}{2} \int_{V} \varepsilon^{\mathrm{T}} \mathbf{E} \varepsilon d V$, where $\varepsilon$ is the strain vector associated with the Green-Lagrange strain tensor and $\mathbf{E}$ is the matrix of elastic coefficients (Sopanen and Mikkola, 2003). The vector of the elastic forces for an element can be defined using the strain energy $U_{s}$ as follows

$$
\mathbf{Q}_{s}=\left(\frac{\partial U_{s}}{\partial \mathbf{e}}\right)^{\mathrm{T}}
$$

The present study is mainly devoted to isotropic, hyperelastic, nonlinear, and nearly incompressible material models, and the proper value of the strain energy density function for these materials is presented in the next Section of the paper.

The mass matrices and vectors of external and elastic forces of the elements follow the standard finite element assembly procedure for each flexible body. In the case of the ANCF, usually all the position and slope coordinates are shared between the elements. Finally, one can write dynamic equations of motion of the constrained flexible multibody system in the general form (Shabana, 2013)

$$
\overline{\mathbf{M}} \ddot{\overline{\mathbf{e}}}+\overline{\mathbf{Q}}_{s}+\boldsymbol{\Phi}_{e}^{\mathrm{T}} \boldsymbol{\lambda}=\overline{\mathbf{Q}}_{e} \quad \boldsymbol{\Phi}=\mathbf{0}
$$

where $\overline{\mathbf{M}}, \overline{\mathbf{Q}}_{s}$ and $\overline{\mathbf{Q}}_{e}$ are, respectively, the mass matrix, the vector of elastic forces and the vector of external forces of the system, $\ddot{\overline{\mathbf{e}}}$ is the acceleration vector of the system, $\boldsymbol{\Phi}$ represents the vector of constraint equations (Sugiyama et al., 2003), $\boldsymbol{\Phi}_{e}=\partial \mathbf{\Phi} / \partial \overline{\mathbf{e}}$ is the Jacobian matrix of constraints and $\boldsymbol{\lambda}$ is the vector of Lagrange multipliers. Equations of motion (2.4) form a set of differential-algebraic equations with the differential index equal to 3 . Finding the solution to these equations is usually a more demanding task than for the solution to ordinary differential equations (Brenan et al., 1996). Moreover, differential-algebraic equations require special numerical techniques, as denoted by Hairer and Wanner (1996). For a review of the 
methods used to solve Eq. (2.4), see e.g. García de Jalón and Bayo (1994). However, the most common methods for solving differential-algebraic equations are the direct integration with, e.g., implicit Runge-Kutta schemes (Hairer and Wanner, 1996), integration of the transformed system with a lower index and stabilization (Gear et al., 1985) or the generalized coordinate partitioning scheme (Wehage and Haug, 1982). In the present work, a Fortran-based research code is used and the implicit Runge-Kutta Radau IIA scheme is utilized. It is worth noting that if all the constrained equations were linear and time independent, system (2.4) would come down to a set of ordinary differential equations $\widehat{\mathbf{M}} \ddot{\hat{\mathbf{e}}}+\widehat{\mathbf{Q}}_{s}=\widehat{\mathbf{Q}}_{e}$ where the vectors and matrices with hat are obtained after linear transformation due to constraint elimination (Garcia-Vallejo et al., 2003).

\subsection{Multi-layer beam models}

Figure 1 presents a two-layer beam model. Two beams, $I$ and $I I$ with local coordinate systems $x_{I} y_{I} z_{I}$ and $x_{I I} y_{I I} z_{I I}$ (for clarity local $z$ axes are omitted) are connected across their height. Each
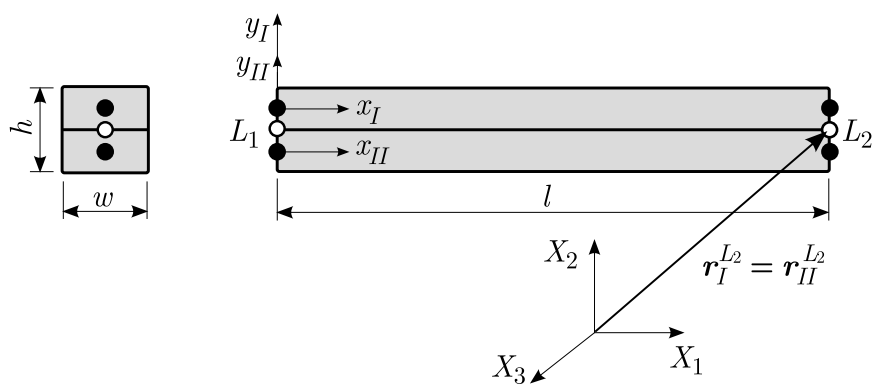

- element nodes

○ layer connectivity points $\left(L_{1}, L_{2}\right)$

Fig. 1. Two-layer beam model

beam has length $l$, width $w$ and height $h / 2$, therefore, the two-beam model has height equal to $h$. The nodes are marked as black dots and they are shared between the elements in each layer, i.e., they follow the standard assembly procedure. The white dots (denoted as $L_{1}$ and $L_{2}$ ) represent the layer connectivity points at which one can impose linear constraint equations between two adjacent elements. $X_{1} X_{2} X_{3}$ is the global reference frame, while the position vectors $\mathbf{r}_{I}^{L_{2}}$ and $\mathbf{r}_{I I}^{L_{2}}$ points to the $L_{2}$ using the parameters, respectively, of elementhe $I$ and $I I$. The layer connectivity constraints can be enforced at the position and slope level, thus the required continuity might be achieved. For example $\boldsymbol{\Phi}^{\mathrm{T}}=\left[\begin{array}{ll}\left(\mathbf{r}_{I}^{L_{1}}-\mathbf{r}_{I I}^{L_{1}}\right)^{\mathrm{T}} & \left(\mathbf{r}_{I}^{L_{2}}-\mathbf{r}_{I I}^{L_{2}}\right)^{\mathrm{T}}\end{array}\right]$ enforces the continuity at the position

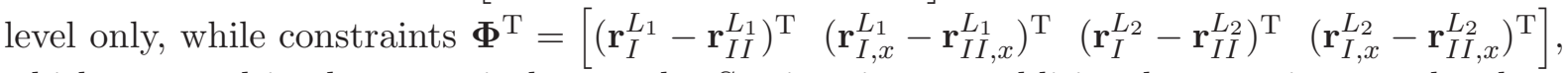
which are used in the numerical examples Section, impose additional constraints on the slope along the local $x$ axis. The total constraint vector should consist of constraints for each layer connectivity point. This approach might be used to build a beam structures with more than two layers across the height or to create a model with layers in both transversal directions. In addition, the use of multi-layer structures further enables the modelling of complex systems like vehicle tires (Patel et al., 2016). As will be shown later in the paper, the multi-layer beam model may ensure a better convergence when the nearly incompressible materials are used. It is worth noting that the approach for modelling a multi-layer beam used by Patel et al. (2016) and introduced in (Liu et al., 2011) is based on subdomains with different material properties created within a single element and cannot be easily adopted for hyperelastic and nonlinear material models. 


\section{Nearly incompressible polynomial material models}

The hyperelastic material models, which are shown in this Section, are in the well-known form of the Mooney-Rivlin models (Bonet and Wood, 1997; Orzechowski and Frączek, 2015). These models are commonly used to represent incompressible rubber-like materials. To fulfil the incompressibility condition, the penalty technique is employed, due to its efficiency and simplicity. Therefore, any member of the Mooney-Rivlin models family can be adopted. Herein, two simplest incompressible models are used and mentioned, namely the Neo-Hookean and twoparameter Mooney-Rivlin.

The strain-energy density function can be written for isotropic materials as a function of the invariants of the deviatoric part of the right Cauchy-Green deformation tensor $\mathbf{C}_{r}=\mathbf{F}^{\mathrm{T}} \mathbf{F}$, defined as $\bar{I}_{1}=J^{-2 / 3} I_{1}$ and $\bar{I}_{2}=J^{-4 / 3} I_{2}$, where $J=\operatorname{det}(\mathbf{F})$ and the invariants of the tensor $\mathbf{C}_{r}$ itself are $I_{1}=\operatorname{tr}\left(\mathbf{C}_{r}\right)$ and $I_{2}=\frac{1}{2}\left[\operatorname{tr}\left(\mathbf{C}_{r}\right)^{2}-\operatorname{tr}\left(\mathbf{C}_{r}^{2}\right)\right]$. In addition, the constraint $J=1$ must be ensured through the body in order to account for the material incompressibility. The strain energy density function in the form of the Mooney-Rivlin models is the following

$$
U_{s}=\sum_{i+j=1}^{K} \mu_{i j}\left(\bar{I}_{1}-3\right)^{i}\left(\bar{I}_{2}-3\right)^{j}
$$

where $\mu_{i j}$ are material coefficients, usually determined from an experiment. $K$ may be an arbitrarily large number, but in practice values of $K>2$ are rarely used. In the present paper, only material models with $K=1$ are considered.

The material models from Eq. (3.1) implicitly assume that the incompressibility is ensured by setting $J$ equal to one. This condition is fulfilled by the penalty method (Maqueda and Shabana, 2007; Orzechowski and Fraczek, 2015). In this technique, the volumetric energy penalty function $U_{p}$ is added to the expression of the strain energy function. This term can be expressed as

$$
U_{p}=\frac{1}{2} k(J-1)^{2}
$$

where $k$ is the penalty coefficient that represent the bulk modulus, a real material property (Bonet and Wood, 1997). In practice, $k$ should be selected sufficiently large to assure incompressibility, but also not too large to avoid numerical complications. The use of energy function from Eq. (3.2) with a finite coefficient $k$ causes that the material can be considered as nearly incompressible only.

Finally, one can combine Eqs (3.1) and (3.2) as

$$
U_{s i c}=U_{s}+U_{p}
$$

To obtain the vector of the elastic forces $\mathbf{Q}_{s}$, the above expression should be integrated over the flexible body volume and inserted into Eq. (2.3). Below, two models based on that representation are shown.

\subsubsection{Incompressible Neo-Hookean material}

The incompressible Neo-Hookean is the simplest member of the Mooney-Rivlin models family, which depends on only one elastic coefficient $\mu_{10}$. Therefore, the expression for the strain energy function can be written as $U_{s}^{n h}=\mu_{10}\left(\bar{I}_{1}-3\right)$, and $\mu_{10}$ is initially equal to one-half of the shear modulus. 


\subsubsection{Incompressible Mooney-Rivlin material}

A two-parameter incompressible Mooney-Rivlin material is another widely used and simple material model which is obtained by assuming that two elastic parameters, $\mu_{10}$ and $\mu_{01}$, are not equal to zero. Therefore, the strain energy density function takes the form $U_{s}^{m r}=\mu_{10}\left(\bar{I}_{1}-3\right)+$ $\mu_{01}\left(\bar{I}_{2}-3\right)$. It can be shown that in the case of small strains, Young's modulus is $E=6\left(\mu_{10}+\mu_{01}\right)$ and the shear modulus is equal to $\mu=2\left(\mu_{10}+\mu_{01}\right)$ (Bathe, 1996).

\section{Locking elimination techniques for nearly incompressible materials}

The locking phenomena can be noticed in the case of many ANCF elements both for linear-elastic (Gerstmayr and Shabana, 2006) and nonlinear (Orzechowski and Frączek, 2015) material models. Its occurrence often causes an erroneously stiff bending characteristic, and is especially noticeable for approaches that directly employ the continuum mechanics. Moreover, a far greater impact of the locking is observed in the case of an incompressible material than in the case of compressible models. The paper presents shortly two methods that can be used for locking suppression for incompressible material models. Both methods were introduced in the previous work (Orzechowski and Fracczek, 2015). In addition, simplifications of the projection space of the $F$-bar method are introduced.

\subsection{Selective reduced integration}

This locking alleviation technique is commonly used to prevent Poisson's locking in many FEA elements (Zienkiewicz and Taylor, 2005) as well as in continuum-based ANCF elements with a linear material model (Gerstmayr et al., 2008). In this method, the integral of the strain energy function is split into two parts that are treated differently. In the first part, which is fully integrated in the total element volume, one does not consider the Poisson effect, while in the second part, which is integrated only along the beam centerline or plate midplane, i.e. uses a reduced integration scheme, one takes the Poisson effect into account. Adequately, the expression of the strain energy density function given by Eq. (3.1) can also be split. The first part of the Mooney-Rivlin material model, denoted as $U_{s}$, can be fully integrated as it does not consider the volumetric effect. However, the volumetric energy penalty function $U_{p}$ should be considered only at the beam axis, as it accounts for the volumetric behaviour. Therefore, the following formula is used

$$
U_{s i c}^{s r i}=\int_{V} U_{s} d V+A \int_{l} U_{p} d l
$$

where $l$ is length of the element, $A$ denotes cross-section area and the index sri designates the selective reduced integration technique.

\section{2. $\quad F$-bar projection method}

The F-bar projection method involves product decomposition of the position vector gradient into volumetric and deviatoric parts (Bonet and Wood, 1997; Elguedj et al., 2008) and is especially convenient to use when the split of the energy density function into deviatoric and volumetric parts is not straightforward and the use of the selective reduced integration might be troublesome. This method is a generalization of the strain projection $B$-bar technique to finite-strain analysis which is considered as an extension of the selective and reduced integration approaches (Hughes, 1987). 
The gradient tensor $\mathbf{F}$ may be split as $\mathbf{F}=\mathbf{F}^{\text {dil }} \mathbf{F}^{\text {dev }}$ where $\mathbf{F}^{\text {dev }}=J^{-1 / 3} \mathbf{F}$ is the deviatoric (volume preserving) part and $\mathbf{F}^{d i l}=J^{1 / 3} \mathbf{I}$ is the volumetric-dilatational part, and $\mathbf{I}$ is the identity matrix. One can notice that $\operatorname{det}\left(\mathbf{F}^{d e v}\right)=1$ and $\operatorname{det}\left(\mathbf{F}^{d i l}\right)=J$. Now, the tensor $\mathbf{F}$ can

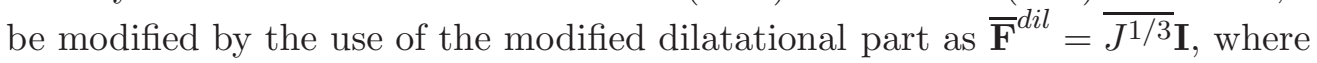

$$
\overline{J^{1 / 3}}=\pi\left(J^{1 / 3}\right)
$$

and $\pi$ is the linear projection operator presented in details below. Consequently, one can write

$$
\overline{\mathbf{F}}=\overline{\mathbf{F}}^{\text {dil }} \mathbf{F}^{\text {dev }}=\frac{\overline{J^{1 / 3}}}{J^{1 / 3}} \mathbf{F}
$$

The modified tensor $\overline{\mathbf{F}}$ can be directly employed to calculate the energy density function given by Eq. (3.1). However, because $U_{s}$ is volume preserving, only the penalty function $U_{p}$ can be affected.

\subsubsection{Projection operator $\pi$}

Studies performed for the isogeometric analysis beam by Elguedj et al. (2008) and further carried out in the ANCF framework by Orzechowski and Fraczek (2015) show that the $L^{2}$ projection of strains is a good candidate for the projection space. Whilst the associated space on which this projection is performed, it should have a constant value in the transversal directions. Therefore, in the previous work (Orzechowski and Frączek, 2015), the following lower-order basis was employed

$$
\widetilde{\mathbf{S}}_{4}=\left[\begin{array}{llll}
1-3 \xi^{2}+2 \xi^{3} & l\left(\xi-2 \xi^{2}+\xi^{3}\right) & 3 \xi^{2}-2 \xi^{3} & l\left(-\xi^{2}+\xi^{3}\right)
\end{array}\right]
$$

where $\widetilde{\mathbf{S}}_{4}$ denotes a lower order cubic basis with four components. However, even a lower order basis may be introduced as constant, linear or quadratic (with one, two and three components, respectively) in the longitudinal direction

$$
\begin{aligned}
& \widetilde{\mathbf{S}}_{1}=[1] \quad \widetilde{\mathbf{S}}_{2}=\left[\begin{array}{ll}
1-\xi & \xi
\end{array}\right] \\
& \widetilde{\mathbf{S}}_{3}=\left[\begin{array}{lll}
2 \xi^{2}-3 \xi+1 & 4 \xi-4 \xi^{2} & 2 \xi^{2}-\xi
\end{array}\right]
\end{aligned}
$$

Next, Eq. (4.2) may be written in the new space (using any basis from Eqs (4.4) and (4.5)) as $\overline{J^{1 / 3}}=\widetilde{\mathbf{S}} \widetilde{\mathbf{J}}^{1 / 3}$ (Elguedj et al., 2008; Hughes, 1987) where

$$
\widetilde{\mathbf{J}}^{1 / 3}=\left[\int_{V} \widetilde{\mathbf{S}}^{\mathrm{T}} \widetilde{\mathbf{S}} d V\right]^{-1} \int_{V} \widetilde{\mathbf{S}}^{\mathrm{T}} J^{1 / 3} d V
$$

The presented procedure corresponds to $L^{2}$ projection of $J^{1 / 3}$ into the $\widetilde{\mathbf{S}}$ basis. Next, the newly calculated value of $\overline{J^{1 / 3}}$ may be substituted into Eq. (3.2) to obtain a modified volumetric penalty function as

$$
\bar{U}_{p}=\frac{1}{2} k\left[\left(\overline{J^{1 / 3}}\right)^{3}-1\right]^{2}
$$

Finally, the strain energy density function for the $F$-bar strain projection method is expressed as follows

$$
U_{s i c}^{F-\text { bar }}=\int_{V} U_{s} d V+A \int_{l} \bar{U}_{p} d l
$$

where $\bar{U}_{p}$ can be calculated only at the element centerline, as the value of $\overline{J^{1 / 3}}$ depends only on the longitudinal coordinate. 


\section{Numerical examples}

Exemplary numerical calculations are carried out with the fully parameterized three-dimensional ANCF beam element with twelve nodal coordinates at each of its two nodes. In order to effectively model bodies with nearly incompressible materials, the techniques of alleviating the volumetric locking presented in Section 4 are applied. In addition, to assembly multi-layer beam structures, the procedure described in Section 2.1 is used. In this study, three simple models of highly deformable clamped beams and physical pendulums are shown. All examples use the standard, spatial, two-node ANCF beam element with twenty-four coordinates (Shabana and Yakoub, 2001; Yakoub and Shabana, 2001).

\subsection{Physical pendulum}

A dynamical analysis of a flexible beam attached at one end to the ground by a spherical joint and falling under the gravity forces is carried out for the purpose of numerical verification. A similar pendulum was examined by Maqueda and Shabana (2007). In the undeformed state, the beam has $1 \mathrm{~m}$ in length, a square cross-section of dimension $20 \mathrm{~mm}$, and a material density of $7200 \mathrm{~kg} / \mathrm{m}^{3}$. In this example, the elastic coefficient for the incompressible Neo-Hookean model is $\mu_{10}=1 \mathrm{MPa}$, while in the case of the two-parameter Mooney-Rivlin material, the values of its coefficients are $\mu_{10}=0.8 \mathrm{MPa}$ and $\mu_{01}=0.2 \mathrm{MPa}$. The material properties allow large deformations of the body. To ensure incompressibility, the penalty term is assumed to be $k=10^{3} \mathrm{MPa}$. The pendulum model is shown in Fig. 2. To increase body deformations, the base of the pendulum is subjected to prescribed motion. The constraint equations for this model can be written as $\boldsymbol{\Phi}^{\mathrm{T}}=\left[\begin{array}{lll}r_{1}^{N}+0.02 \sin (2 \pi t) & r_{2}^{N} & r_{3}^{N}\end{array}\right]$, where $r_{i}^{N}$ for $i=1,2,3$ denotes the component of the position vector of the node $N$ (in meters), and $t$ is time expressed in seconds.
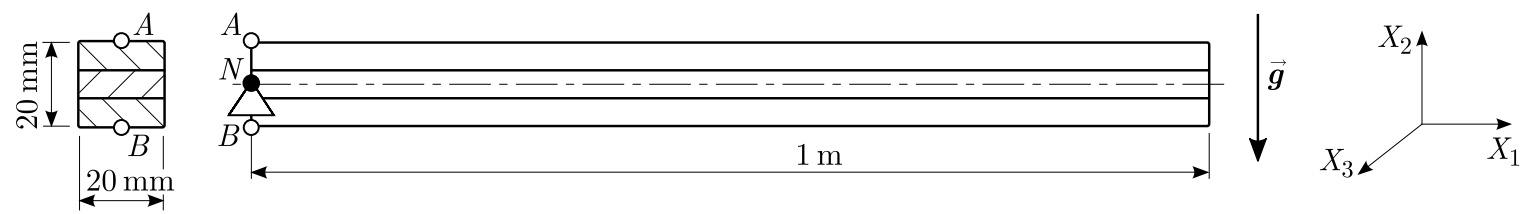

Fig. 2. Very flexible physical pendulum, three-layer model

The pendulum body is built of four beam elements along its length. Figures $3 \mathrm{a}$ and $3 \mathrm{~b}$ show displacements of the pendulum free end tip resulting from performed dynamical simulations for three types of models: without the locking suppression method, with the $F$-bar projection and the three-layer model with the $F$-bar projection. Figure 3 a presents results for the NeoHooken material, while in Fig. 3b results for the Mooney-Rivlin material are shown. Despite the differences in materials, the results in both figures are very similar. The models without the locking suppression method show very small deformations as they behave almost like a rigid body, despite very low elastic coefficients and large pendulum density. On the other hand, all models that employ the locking elimination by the $F$-bar projection show reasonable deformations. The lack of the over-stiff response in the incompressible models during bending indicates that the influence of the volumetric locking has been suppressed (Orzechowski and Fracczek, 2015). Therefore, it can be concluded that the influence of the volumetric locking on the results of hyperelastic nearly incompressible material models might be enormous, and that the multi-layer beam model produces acceptable results, comparable with those of the standard beam model. The results for the selective reduced integration exhibit very similar behaviour.

Figures $4 \mathrm{a}$ and $4 \mathrm{~b}$ show how well the incompressibility condition is preserved by the analysed models by plotting the value of the determinant of the deformation gradient tensor $J=\operatorname{det}(\mathbf{F})$. Figure 4a presents the value of $J$ as a function of time at the point $A$ of the body (see Fig. 2). 

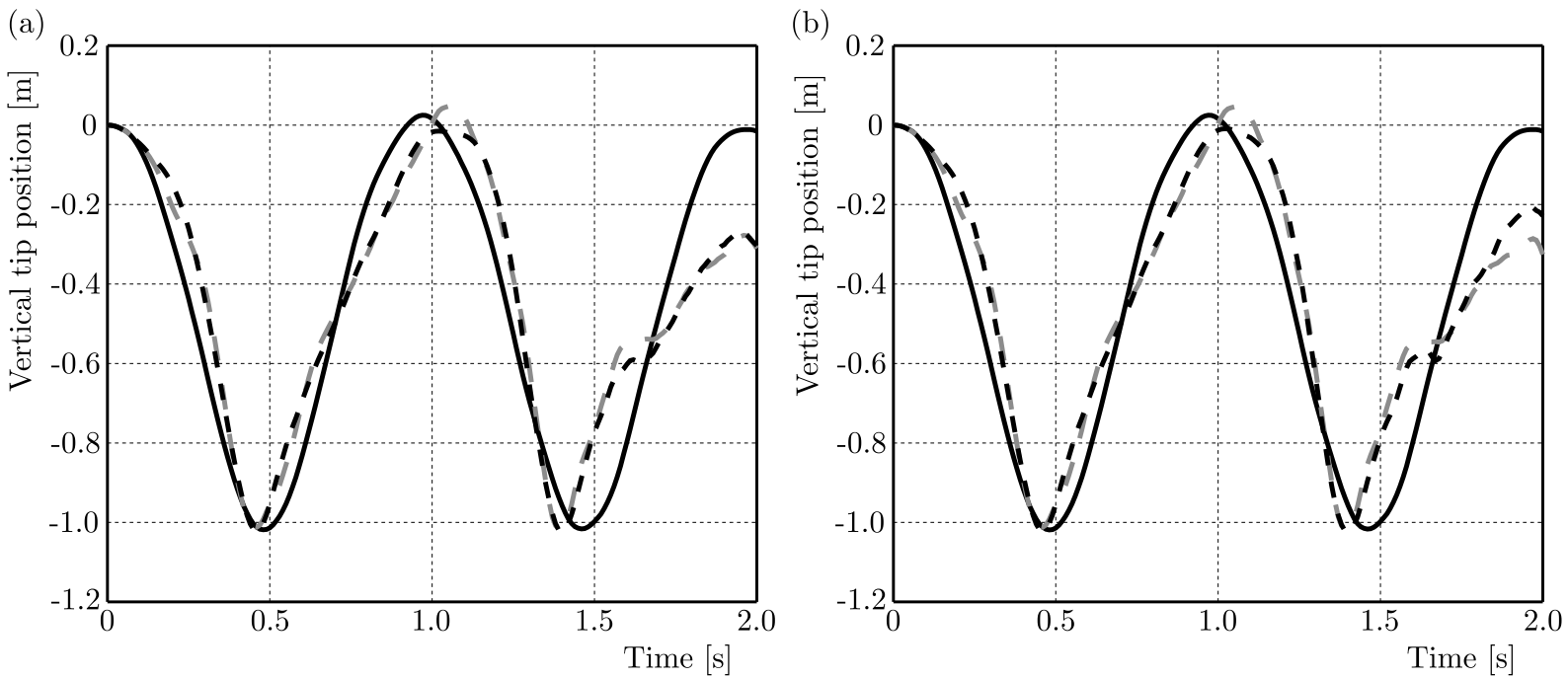

Fig. 3. Vertical position of the beam free end tip for (a) incompressible Neo-Hookean and

(b) incompressible Mooney-Rivlin material: (-) without the locking suppression method, ( $\square$ ( $)$ with the $F$-bar projection, (- - - $)$ ) with the $F$-bar projection, three-layer model

(a)

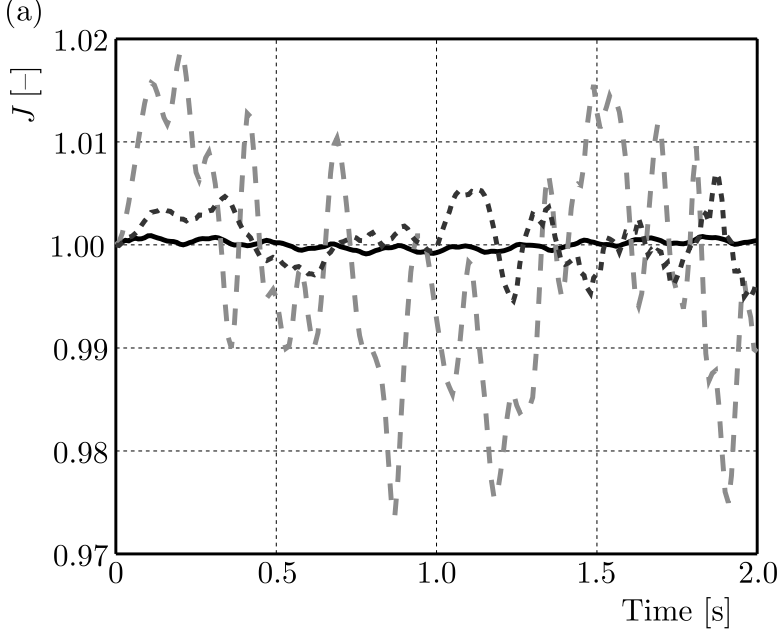

(b)

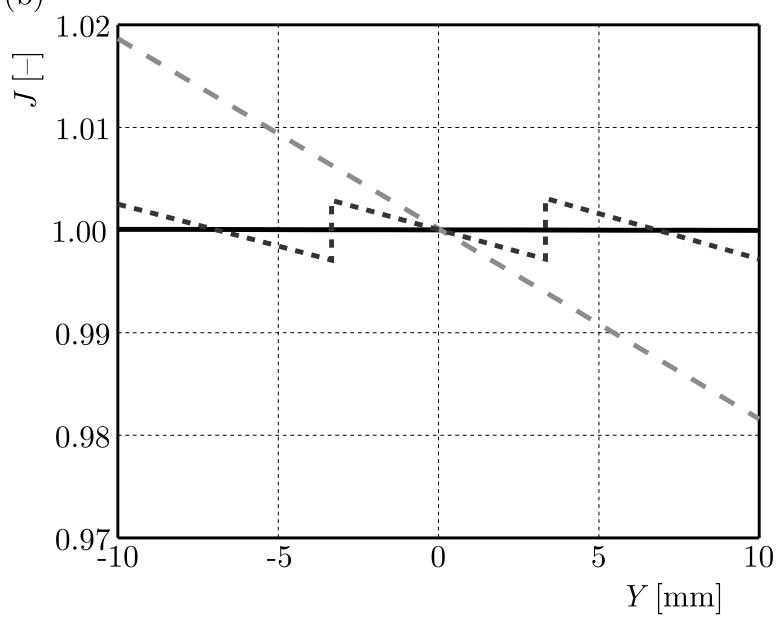

Fig. 4. Value of $J=\operatorname{det}(\mathbf{F})$ (a) in time and (b) along beam thickness for incompressible Neo-Hookean

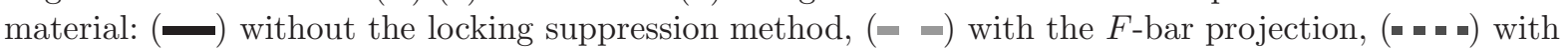
the $F$-bar projection, three-layer model

Likewise, Fig. 4b presents the value of $J$ along the line connecting points $A$ and $B$ of the body (shown in Fig. 2) for a specific time instant (at $t=0.2 \mathrm{~s}$ ). It can be clearly seen that the incompressibility condition for the whole body volume is preserved only for the formulation that does not use any locking alleviation technique.

For beams using the $F$-bar strain projection method, the value of $J$ is changing noticeably, however, in the case of the three-layer model this violation is significantly smaller than for the one-layer beam as the region of constraint violation is bounded to the beam boundaries. Since the lack of continuity of the $J$ value between the beam layers is crucial for preventing the locking behaviour (continuous $J$ would result in its constant value equal to one), it must be ensured that the continuity does not occur on the gradient perpendicular to the layer boundary.

\subsection{Cantilever beam}

In this example, static simulation of the cantilever beam, similar to that shown in Figure 5, is employed. The beam is clamped at one end with the help of a linear constraint equation in 
the form of $\boldsymbol{\Phi}=\mathbf{e}^{N}$. The gravitational force is the only external force that acts on the system. The material and geometrical properties of the flexible body are the same as in the previous example, except for the size of the cross section that is increased to $40 \mathrm{~mm}$ for both height and width. For a verification purpose, the ANCF models with one, two and three layers are examined and the results of simulations are compared with the solution of an analogous model obtained with a commercial FEA package (ANSYSR Academic Research, 2010).
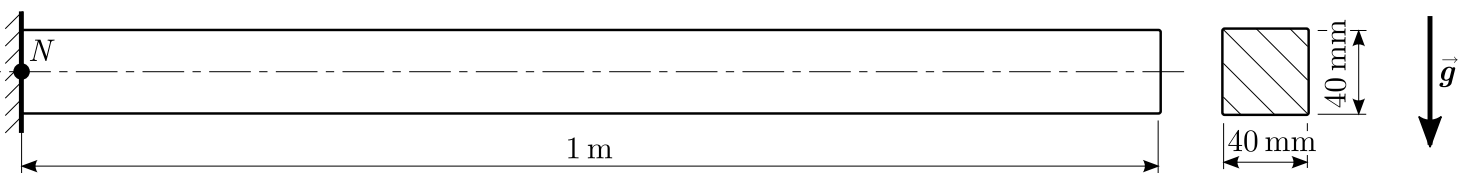

Fig. 5. Very flexible cantilever beam under gravity forces

The main purpose of this example is to assess the impact of the new modelling techniques, like the use of the multi-layer beams and the low order projection basis, on the obtained solution. The results are shown for both presented locking elimination techniques, the $F$-bar strain projection and the selective reduced integration as well as for both material models, the incompressible NeoHookean and two-parameter Mooney-Rivlin. For the F-bar method, four different projection bases are employed as has been proposed in Section 4.2. The results are compared against the FEA package solution. For this reason, we applied a very fine mesh in the FEA package consisting of 1600 higher-order solid elements (SOLID186).

In Table 1, the results of static ANCF analysis are presented for the models with one, two and three layers across thickness, together with reference FEA results. The results show for both locking elimination procedures, the SRI and $F$-bar, the convergence to nearly the same solution for a given number of layers and irrespective of the employed projection basis.

Table 1. Deformation of the free end tip of the clamped beam for models with 48 elements in each layer (NoL - number of layers, ICNH - incompressible Neo-Hookean, ICMR - incompressible Mooney-Rivlin, SRI - selective reduced integration). Results for one-layer models for SRI and cubic $F$-bar locking suppression methods are taken from (Orzechowski and Frączek, 2015)

\begin{tabular}{|l|c|c|c|c|c|}
\hline \multirow{2}{*}{ Method } & \multirow{2}{*}{ NoL } & \multicolumn{2}{|c|}{ ICNH analysis } & \multicolumn{2}{c|}{ ICMR analysis } \\
\cline { 3 - 6 } & & $\mathrm{X}[\mathrm{m}]$ & $\mathrm{Y}[\mathrm{m}]$ & $\mathrm{X}[\mathrm{m}]$ & $\mathrm{Y}[\mathrm{m}]$ \\
\hline \hline FEA & - & -0.828 & -0.940 & -0.828 & -0.941 \\
\hline \multirow{2}{*}{ ANCF SRI } & 1 & -0.894 & -0.965 & -0.893 & -0.964 \\
& 2 & -0.838 & -0.941 & -0.838 & -0.941 \\
& 3 & -0.829 & -0.938 & -0.830 & -0.938 \\
\hline \multirow{2}{*}{ ANCF F-bar constant } & 1 & -0.894 & -0.965 & -0.893 & -0.965 \\
projection basis & 2 & -0.840 & -0.943 & -0.840 & -0.943 \\
& 3 & -0.832 & -0.940 & -0.832 & -0.941 \\
\hline \multirow{2}{*}{ ANCF F-bar linear } & 1 & -0.894 & -0.965 & -0.893 & -0.965 \\
projection basis & 2 & -0.837 & -0.941 & -0.838 & -0.941 \\
& 3 & -0.829 & -0.938 & -0.830 & -0.939 \\
\hline \multirow{2}{*}{ ANCF F-bar quadratic } & 1 & -0.892 & -0.963 & -0.891 & -0.962 \\
projection basis & 2 & -0.837 & -0.941 & -0.838 & -0.941 \\
& 3 & -0.829 & -0.938 & -0.830 & -0.938 \\
\hline \multirow{2}{*}{ ANCF F-bar cubic } & 1 & -0.891 & -0.962 & -0.890 & -0.962 \\
projection basis & 2 & -0.837 & -0.941 & -0.838 & -0.941 \\
& 3 & -0.829 & -0.938 & -0.830 & -0.938 \\
\hline
\end{tabular}


The results presented in Table 1 show that the convergent solutions given by ANCF are noticeably different when different numbers of layers are used. For the one-layer model, the ANCF results are significantly different from the reference. The noticeable discrepancy between the reference FEA results and the ANCF models is mainly due to violation of the incompressibility assumption at the beam surface. Nevertheless, this behaviour is consistent with the characteristics of the finite element analysis, where incompressibility is ensured only on the average (Adler et al., 2014). The standard approach to deal with such a problem is to employ a larger number of elements across thickness, and the results in Table 1 show that the beam models with two and three layers conform much better to the reference. Therefore, when the incompressible material model employs the locking elimination technique based on reduced integration, it is crucial to apply more elements across the transversal direction in order to obtain reasonable results. In addition, in the case of the $F$-bar method, the simplest constant projection basis is able to reproduce a proper solution that is only slightly different than the reference one, while offering the smallest computational complexity.

\subsection{Cantilever rubber-like beam}

The last example is the dynamic analysis of the clamped beam model, wherein the constraint equations are identical as those in the previous Section. The system is similar to the model shown by Jung et al. (2011). The body undergoes the gravitational force, and is made of a rubber-like material having density of $2150 \mathrm{~kg} / \mathrm{m}^{3}$, Kirchhoff's modulus of $1.91 \mathrm{MPa}$ and the penalty term of $10^{3} \mathrm{MPa}$. The beam has a rectangular cross section of $7 \mathrm{~mm}$ width and $5 \mathrm{~mm}$ height, and $0.35 \mathrm{~m}$ in length. The models use thirty fully parameterized ANCF beam elements for each layer, whilst one- and three-layer models are compared. Such a large number of elements is needed to obtain a converged solution for both locking elimination procedures. The results of ANCF simulations are compared with the results obtained with the FEA package. The beam elements from the classic FEA package cannot be used with nonlinear incompressible material models. Therefore, SOLID185 (ANSYS@ Academic Research, 2010) elements with reduced integration are used. To obtain a convergent solution, the use of 336 solids is sufficient. In this example, the only considered material model is the incompressible Neo-Hookean.
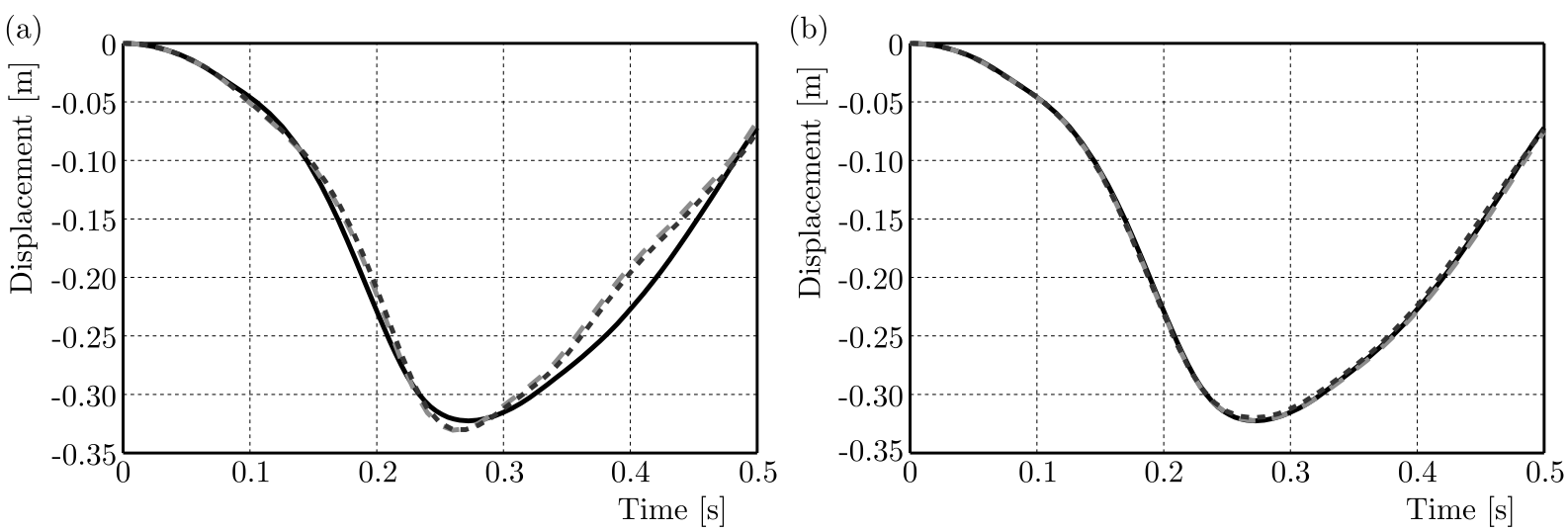

Fig. 6. Beam end tip displacements, (a) one-layer model, (b) three-layer model: ( $\mathbf{a}$ ) reference FEA, $(-\square)$ ANCF with selective reduced integration, (-m) ANCF full integration with $\overline{\mathbf{F}}$

Figure 6a shows the results for two ANCF models with different locking suppression methods and for the reference FEA solution (Orzechowski and Frączek, 2015). The results are recalled here for easier comparison with the three-layer model. Both ANCF models provide almost the same results, and for the one-layer model a reasonably good agreement with the reference can be observed. However, the results in Fig. 6b show a large improvement for the three-layer model, irrespective of the employed projection basis. The results for the SRI present a similar advance. 
One can conclude that the alleviation of the volumetric locking effect and introduction of the multi-layer structures leads to a substantial improvement as compared with the one-layer model and ensures the quality of the results obtained using higher-order beam elements with forty-two nodal parameters as shown by Orzechowski and Frączek (2015). In addition, in comparison with the FEA solid model, the number of parameters involved is, in the given application, more than four times less in the ANCF one-layer beam model, and two times less in the case of the three-layer model.

\section{Conclusions}

The ANCF is used successfully in the analysis of flexible bodies undergoing large deformations. The current study presents that nonlinear and nearly incompressible material models can be included in the ANCF in a straightforward way. The implementation of nonlinear incompressible materials and locking elimination techniques within the ANCF framework presented so far in the literature exhibit inaccurate behaviour in bending-dominated examples for a standard twenty-four parameter three-dimensional beam element.

In order to carry out accurate and efficient model simulations with incompressibility, two locking elimination techniques are presented - the $F$-bar strain projection method and the selective reduced integration. Both methods are implemented in the ANCF framework and tested with multi-layer beam structures. In addition, for the $F$-bar method, different projection spaces are investigated. Numerical tests show that the locking influence for an incompressible material models is enormous. The results of ANCF simulations are compared with a commercial FEA package and a very good agreement is found, especially for three-layer models. In the case of the $F$-bar method, the order of the projection space has a minor influence on the quality of the solution.

In the classical FEA, solid elements must be used when the materials with a nonlinear characteristic are used to model slender structures. In contrast, in the ANCF, fully parameterized beam elements can be employed in such systems, the result of which are models with much less parameters than for the FEA. However, the application of ANCF beams modelled with nonlinear materials to complex cross-sectional shapes might require further investigations (Orzechowski, 2012; Orzechowski and Shabana, 2016).

A desirable direction for future research is the development of alternative methods that would enable efficient locking elimination. In addition, it is indicated in the literature that, applying the systems which use incompressible material models, one may obtain solutions that are highly imprecise in stresses, although a proper solution exist for displacements (Bathe, 1996). Hence, the distribution of stresses in such models should also be taken into account as an essential research topic.

\section{Acknowledgements}

This work has been supported under grant No. DEC-2012/07/B/ST8/03993 by the National Science Centre.

\section{References}

1. Adler J.H., Dorfmann L., Han D., Maclachlan S., Paetsch C., 2014, Mathematical and computational models of incompressible materials subject to shear, IMA Journal of Applied Mathematics, 79, 5, 889-914

2. ANSYS@ACADEmic Research, 2010, ANSYS Mechanical APDL Documentation, ANSYS Inc., release 13.0 
3. Bathe K.J., 1996, Finite Element Procedures, Prentice Hall, New Jersey

4. Bonet J., Wood R.D., 1997, Nonlinear Continuum Mechanics for Finite Element Analysis, Cambridge University Press, Cambridge; New York, NY, USA

5. Brenan K.E., Campbell S.L., Petzold L.R., 1996, Numerical Solution of Initial-Value Problems in Differential-Algebraic Equations, SIAM, Philadelphia

6. Elguedj T., Bazilevs Y., Calo V.M., Hughes T.J., 2008, B-bar and F-bar projection methods for nearly incompressible linear and non-linear elasticity and plasticity using higher-order NURBS elements, Computer Methods in Applied Mechanics and Engineering, 197, 33-40, 2732-2762

7. García de Jalón J., Bayo E., 1994, Kinematic and Dynamic Simulation of Multibody Systems. The Real-Time Challenge, Springer, New York

8. Garcia-Vallejo D., Escalona J.L., Mayo J., Domínguez J., 2003, Describing rigid-flexible multibody systems using absolute coordinates, Nonlinear Dynamics, 34, 1-2, 75-94

9. Gear C.W., Leimkuhler B., Gupta G.K., 1985, Automatic integration of Euler-Lagrange equations with constraints, Journal of Computational and Applied Mathematics, 12-13, 77-90

10. Gerstmayr J., Matikainen M.K., Mikkola A.M., 2008, A geometrically exact beam element based on the absolute nodal coordinate formulation, Multibody System Dynamics, 20, 4, 359-384

11. Gerstmayr J., Shabana A.A., 2006, Analysis of thin beams and cables using the absolute nodal co-ordinate formulation, Nonlinear Dynamics, 45, 1, 109-130

12. Hairer E., Wanner G., 1996, Solving Oridinary Differential Equations II. Stiff and Differential-Algebraic Problems, Springer, Berlin, 2nd edition

13. Hughes T.J.R., 1987, The Finite Element Method. Linear Static and Dynamic Finite Element Analysis, Prentice Hall, New Jersey

14. Jung S., PArk T., Chung W., 2011, Dynamic analysis of rubber-like material using absolute nodal coordinate formulation based on the non-linear constitutive law, Nonlinear Dynamics, 63, $1,149-157$

15. Liu C., Tian Q., Hu H., 2011, Dynamics of a large scale rigid-flexible multibody system composed of composite laminated plates, Multibody System Dynamics, 26, 3, 283-305

16. Maqueda L.G., Mohamed A.N.A., Shabana A.A., 2010, Use of general nonlinear material models in beam problems: Application to belts and rubber chains, Journal of Computational and Nonlinear Dynamics, 5, 2, 021003, 10 pages

17. Maqueda L.G., Shabana A.A., 2007, Poisson modes and general nonlinear constitutive models in the large displacement analysis of beams, Multibody System Dynamics, 18, 3, 375-396

18. Orzechowski G., 2012, Analysis of beam elements of circular cross section using the absolute nodal coordinate formulation, Archive of Mechanical Engineering, LIX, 3, 283-296

19. Orzechowski G., Frączek J., 2015, Nearly incompressible nonlinear material models in the large deformation analysis of beams using ANCF, Nonlinear Dynamics, 82, 1, 451-464

20. Orzechowski G., Shabana A.A., 2016, Analysis of warping deformation modes using higher order ANCF beam element, Journal of Sound and Vibration, 363, 428-445

21. Patel M., Orzechowski G., Tian Q., Shabana A.A., 2016, A new multibody system approach for tire modeling using ANCF finite elements, Proceedings of the Institution of Mechanical Engineers, Part K: Journal of Multi-body Dynamics, 230, 1, 69-84

22. Shabana A.A., 1997a, Definition of the slopes and the finite element absolute nodal coordinate formulation, Multibody System Dynamics, 1, 3, 339-348

23. Shabana A.A., 1997b, Flexible multibody dynamics: review of past and recent developments, Multibody System Dynamics, 1, 2, 189-222

24. Shabana A.A., 2008, Computational Continuum Mechanics, Cambridge University Press, Cambridge, first edition 
25. Shabana A.A., 2013, Dynamics of Multibody Systems, Cambridge University Press, 4th edition

26. Shabana A.A., Yakoub R.Y., 2001, Three dimensional absolute nodal coordinate formulation for beam elements: theory, Journal of Mechanical Design, 123, 4, 606-613

27. Sopanen J.T., Mikkola A.M., 2003, Description of elastic forces in absolute nodal coordinate formulation, Nonlinear Dynamics, 34, 1, 53-74

28. Sugiyama H., Escalona J.L., Shabana A.A., 2003, Formulation of three-dimensional joint constraints using the absolute nodal coordinates, Nonlinear Dynamics, 31, 2, 167-195

29. Sugiyama H., Gerstmayr J., Shabana A.A., 2006, Deformation modes in the finite element absolute nodal coordinate formulation, Journal of Sound and Vibration, 298, 4-5, 1129-1149

30. Wasfy T.M., Noor A.K., 2003, Computational strategies for flexible multibody systems, Applied Mechanics Reviews, 56, 6, 553-613

31. Wehage R.A., Haug E.J., 1982, Generalized coordinate partitioning for dimension reduction in analysis of constrained dynamic systems, Journal of Mechanical Design, 104, 1, 247-255

32. Yakoub R.Y., Shabana A.A., 2001, Three dimensional absolute nodal coordinate formulation for beam elements: implementation and applications, Journal of Mechanical Design, 123, 4, 614-621

33. Zienkiewicz O.C., TAYlor R.L., 2005, The Finite Element Method for Solid and Structural Mechanics, Butterworth-Heinemann, Oxford, 6th edition

Manuscript received September 19, 2016; accepted for print April 5, 2017 